\title{
Universidad y desarrollo sostenible
}

\author{
University and Sustainable Development
}

\begin{abstract}
David Vallespín Pérez
Catedrático de Derecho Procesal

Departamento de Derecho Administrativo, Derecho Procesal y Derecho Financiero y Tributario

Universidad de Barcelona

E-mail: dvallespin@ub.edu
\end{abstract}

\begin{abstract}
Resumen: El presente artículo tiene por objetivo analizar el papel decisivo que corresponde jugar a la universidad pública en cuanto al cumplimiento de los Objetivos de Desarrollo Sostenible contemplados en la Agenda 2030. Concretamente, desde la perspectiva del Objetivo Número 4 (Educación de Calidad), centraremos nuestra atención en el libre acceso a la educación superior y el papel de ascensor social que corresponde a la universidad; el diseño de un nuevo contrato social fundado, en buena medida, en el cumplimiento, bajo la cultura de la calidad, de las misiones universitarias; la política medioambiental que corresponde desarrollar a las instituciones de educación superior; la igualdad efectiva entre mujeres y hombres en el ámbito universitario; y la configuración de la universidad como espacio de integración y gestión de la diversidad, integrador y saludable.
\end{abstract}

Palabras clave: Desarrollo sostenible, Agenda 2030, Universidad, calidad docente, protección medioambiental, igualdad de género, diversidad, cohesión social. 
Abstract: The aim of this article is to analyse the decisive role that public universities have to play in meeting the sustainable development objectives set out in Agenda 2030. Specifically, from the perspective of Objective 4 (Quality Education), we will focus our attention on free access to higher education and the role of the university as a social lift; the design of a new social contract based, to a large extent, on the fulfilment, under the culture of quality, of university missions; the environmental policy that corresponds to the development of higher education institutions; effective equality between men and women in the university environment; and the configuration of the university as a space for integration and management of diversity, inclusive and healthy.

Keywords: Sustainable development, 2030 Agenda, University, Teaching quality, Environmental protection, Gender equality, Diversity, Social cohesion.

Sumario: 1. El papel de la universidad ante el desarrollo sostenible (planteamiento general). 2. El "libre acceso" a la universidad pública como garantía de su papel como "ascensor social". 3. La universidad ante el "nuevo contrato social". La "cultura de la calidad" en el cumplimiento de las misiones universitarias. 4. Política medioambiental y universidad. 5. La igualdad efectiva entre mujeres y hombres en el ámbito universitario. 6. Universidad integradora, saludable e implicada en la gestión de la diversidad y la cohesión social. 7. Conclusiones. 8. Bibliografía.

\section{El papel de la universidad ante el desarrollo sostenible (planteamiento general)}

La política universitaria, en pleno siglo XXI, debe fundarse en un claro e inequívoco compromiso para con el desarrollo sostenible, concebido éste, como así se recogió en el Informe Brundtland de 1987, como aquél que satisface las necesidades del presente sin comprometer las de las generaciones futuras (SUREDA, SÁNCHEZ y BENAYAS, 9 de junio de 2017, párr. 2). La sostenibilidad giró, inicialmente, sobre tres grandes pilares: el económico, el social y el ambiental. Ello es así, porque sus objetivos tenían que ver con la eficiencia económica, la inclusión social y el respeto medioambiental.

Años más tarde, en la Cumbre de la Tierra celebrada en Río de Janeiro, en 1992, el desarrollo sostenible adquirió un impulso global mediante la llamada Declaración de Río sobre Medio Ambiente y Desarrollo, la creación de la Convención Marco de Naciones 
Unidas sobre el cambio climático, la ratificación de los convenios sobre Bosques y Biodiversidad y la adopción de la Agenda 2030. Una década más tarde, con la celebración en el año 2012 de la Conferencia sobre Desarrollo Sostenible de Río+20, se incorporó un cuarto pilar a la sostenibilidad: cultura y educación.

De hecho, la aprobación de la Agenda 2030 en 2015 implicó la individualización de los 17 Objetivos de Desarrollo Sostenible (en adelante ODS): finalización de la pobreza; hambre cero; salud y bienestar; educación de calidad; igualdad de género; agua limpia y saneada; energía asequible y no contaminante; trabajo digno y crecimiento económico; industria e innovación; reducción de desigualdades; ciudades y comunidades sostenibles; producción y consumo responsables; acción por el clima; vida submarina; vida de ecosistemas terrestres; paz, justicia e instituciones sólidas; y alianza mundial orientada a alcanzar un auténtico y genuino desarrollo sostenible.

Siendo esto así, las universidades, mediante la generación de conocimiento, están llamadas a ayudar de manera directa e indirecta a la consecución efectiva de dichos objetivos de desarrollo sostenible (FACHELLI, 2019, 21-22). Su implicación directa con algunos objetivos es patente (Objetivo 4 - Educación de Calidad), pero también lo es que, indirectamente, las instituciones de educación superior también contribuyen al respeto de todos y cada uno de los demás ODS (GARCÍA BENAU, 14 de enero de 2019, párr. 4).

Para conseguir hacer efectivos dichos objetivos, las diferentes administraciones, más allá de las buenas palabras o "brindis al sol”, deben ser conscientes que la universidad precisa de un nuevo modelo de financiación (OWENS, LENNARD y ANDRÉS AUCEJO, 2020, 3-7). Modelo éste inaplazable (VALLESPÍN PÉREZ, 29 de mayo de 2018, párrs. 1-2) y en el que también es obligado tomar en consideración la necesidad de afrontar la transformación digital de la universidad (VALLESPÍN PÉREZ, 29 de mayo de 2018, párr. 2).

Un modelo que se sustente, en primer lugar, en una financiación basal, suficiente, estable y que permita hacer frente a los costes básicos de la docencia, la investigación, la transferencia e innovación y la gestión, así como mantener y mejorar las infraestructuras y equipamientos universitarios; en segundo lugar, en la negociación de un complemento de financiación pública por la vía del contrato-programa definido en función del 
cumplimiento de determinados objetivos; y, en tercer y último lugar, por la activación de diferentes vías de fundraising que permitan, siempre dejando a salvo la autonomía universitaria, captar fondos de naturaleza privada que puedan servir para poner en marcha nuevos proyectos, así como aprovechar nuevas oportunidades de investigación en un mundo cambiante (VALLESPÍN PÉREZ, 24 de octubre de 2019, párrs. 5-7).

Dado lo anterior, la universidad, como institución, debe liderar la sociedad con compromiso, así como defender el papel fundamental que corresponde al conocimiento, la concienciación ética y la sostenibilidad (RODRÍGUEZ LOZANO, 23 de junio de 2020, párrs. 4-6). Más concretamente, como así se ha podido constatar en la Cumbre de Madrid de 2019, la universidad no solo debe cumplir sus funciones con calidad, sino también respetar la igualdad de oportunidades, contribuir a la reducción de la "huella planetaria" a nivel ecológico, y fomentar un uso responsable y sostenible de los recursos naturales ${ }^{119}$.

\section{El "libre acceso" a la universidad pública como garantía de su papel como "ascensor social"}

Las y los estudiantes universitarios constituyen la razón de ser de la universidad. Son las actrices y actores clave de la actividad formativa que es propia de la universidad. Su satisfacción, en cuanto presente y futuro de la sociedad, es un claro indicador acerca de la calidad de cualquier sistema universitario. En consecuencia, el acceso a la educación superior no puede concebirse nunca como un privilegio, sino antes, al contrario, como un auténtico derecho (VALLESPÍN PÉREZ, 18 de octubre de 2016, párr. 2).

De este modo, la universidad pública seguirá cumpliendo, como no puede ser de otra forma en un Estado social y democrático de Derecho, con su misión de "ascensor social" fundada, exclusivamente, en el mérito y capacidad de las personas, esto es, en la justa valoración, en positivo, de la cultura del sacrificio.

\footnotetext{
119 Acerca del papel de "palanca de cambio" social que cabe atribuir a los ODS y la Cuarta Revolución Industrial, véase, por todos: GARCÍA, I. y ASTIGARRAGA, E. (1 de mayo de 2020). Los objetivos de desarrollo sostenible en la formación universitaria. Blog de Studia XXI - Universídad. Recuperado de: https://www.universidadsi.es/objetivos-de-desarrollo-sostenible-universidad/. Sobre la estrecha relación entre desarrollo sostenible y universidad, especialmente en el terreno de las ciencias experimentales, véase: VALLESPÍN PÉREZ, D. (17 de junio de 2019). Desarrollo sostenible, biogeología y universidad. Blog de Studia XXI - Universídad. Recuperado de: https://www.universidadsi.es/desarrollo-sosteniblebiogeologia-y-universidad/.
} 
De conformidad con los ODS es obligado defender una universidad pública accesible para todos. Es necesario, por tanto, adoptar todas aquellas medidas que garanticen el principio de igualdad de oportunidades en orden al acceso y continuidad en todos los niveles formativos de la educación superior. En esta dirección, por ejemplo, cabe situar todo aquello que tiene que ver con la reducción de las tasas universitarias y el diseño de un completo sistema de becas y ayudas que permitan hacer posible que ningún estudiante pueda quedar excluido, por motivos económicos o sociales, de la universidad pública de calidad (VALLESPÍN PÉREZ, 5 de octubre de 2020, párrs. 6-7).

Las universidades, máxime en una situación presidida por la crisis económica, sanitaria y social derivada de la pandemia del coronavirus, deberán mejorar y facilitar acciones que permitan flexibilizar los pagos de las matrículas en diferentes mensualidades. Igualmente, los diferentes gobiernos deberán diseñar, en paralelo, un sistema de becas general en el que no solo tenga cabida la recuperación de la llamada beca-salario, sino también la de aquellas referidas a la compra de libros, material y transporte.

El plan de becas integral deberá mejorar en cantidad y calidad, fundándose en criterios sociales y económicos, la movilidad geográfica, los resultados académicos y el costeoportunidad de los estudios superiores. Junto a dicho plan también será oportuno prestar la suficiente atención a las becas-idiomas, los programas propios de ayuda al estudio de las propias instituciones universitarias, y las becas de tecnología o conectividad, pues no podemos obviar la constatación de la denominada "brecha digital".

\section{La universidad ante el "nuevo contrato social". La "cultura de la calidad" en el cumplimiento de las misiones universitarias}

Las universidades, en pleno siglo XXI, deben saber aprovechar la voluntad y necesidad de priorizar el conocimiento para posicionarse como actores claves de un nuevo ecosistema de conocimiento orientado a hacer posible la mejora de la calidad de vida y bienestar de la sociedad. La aspiración de vivir en un mundo mejor, digitalmente inteligente, con una economía de conocimiento sólida y en la que todas las personas puedan gozar de una auténtica igualdad de oportunidades, nos sitúa ante la necesidad, 
inaplazable, de afrontar la transformación del sistema universitario (VALLESPÍN PÉREZ, 15 de marzo de 2020, párr. 2).

Cualquier institución universitaria debe proyectar su futuro sobre la base del reconocimiento de sus puntos fuertes, pero también tomando plena consciencia de sus necesidades y problemas. La universidad, como es lógico, debe adaptarse a las nuevas exigencias sociales. En consecuencia, no debe limitarse, sin más, a transmitir el saber, sino que también debe generar opinión, así como demostrar su claro compromiso con el progreso social y su entorno ${ }^{120}$. La universidad debe educar a personas intelectualmente maduras y comprometidas con la sociedad. De ahí la importancia, precisamente, de poner en valor la función social e integradora de la universidad en el marco de construcción, consolidación y evolución de la sociedad del conocimiento.

La formación, el talento, la educación, la ciencia, la tecnología, la innovación y la cultura emprendedora están llamadas a generar "beneficios para la ciudadanía”. En una sociedad, como la actual, en la que ha calado una sensación de malestar y desconfianza en aquellas personas que se sienten desprotegidas por la globalización, injustamente tratadas por la economía de mercado, temerosas ante la migración, abandonadas en áreas rurales, carentes de cobertura social, desamparadas ante la transformación digital y el cambio tecnológico, alarmadas por el cambio climático y sus consecuencias, o sometidas a las incertezas del nuevo mundo que ha nacido por obra y gracia del efecto de la Covid-19; ha llegado la hora de afrontar el diseño de un "nuevo contrato social" ${ }^{121}$. Un contrato en el que la universidad, al amparo de los Objetivos de Desarrollo Sostenible de la Agenda 2030, debe asumir un papel protagonista que haga posible, pese a las dificultades del momento presente, que toda persona esté en condiciones de "vivir una vida digna de ser vivida".

La universidad no puede abstenerse de asumir un rol principal en orden a la construcción de una sociedad abierta, dialogante, plural, educada, integradora, culta y conectada a nivel

\footnotetext{
${ }^{120}$ Para una correcta comprensión de la rendición de cuentas que es propia de la responsabilidad social de las universidades, véase: MONEVA ABADÍA, J.M. y MARTÍN VALLESPÍN, E. (2012). Universidad y Desarrollo Sostenible: análisis de la rendición de cuentas de las universidades públicas desde un enfoque de responsabilidad social. Revista iberoamericana de contabilidad de gestión, 10 (19), 1-19.

${ }^{121}$ Para un estudio completo acerca de lo que supone este nuevo contrato social, véase, por todos: COSTAS, A. (2017). El final del desconcierto. Un nuevo contrato social para que España funcione. Madrid: Atalaya, 303-321.
} 
internacional; la configuración de un tejido productivo innovador y basado en la investigación y la transferencia de conocimiento; y la consolidación de una mayor y mejor cohesión social. En consecuencia, la universidad, en su dimensión de institución justa, social y democrática, así como en el contexto de la sociedad del conocimiento, debe convertirse en uno de aquellos motores que hagan posible alcanzar una riqueza inteligente, solidaria y sostenible (VALLESPÍN PÉREZ, 21 de mayo de 2020, párr. 5).

Siento esto así, entre las prioridades propias de la denominada Agenda Educativa Común (Agenda 2030) las universidades deben apostar por una política docente integradora, equitativa, de calidad, con implicación social, que fomente el aprendizaje científico, técnico, social, artístico y cultural, y orientada a la transferencia de conocimiento y la inserción laboral.

Una política académica y docente, incardinada en una universidad global e internacional, que deberá partir de una racionalización del actual mapa de titulaciones, la creación de grados y dobles titulaciones de calidad, presididos por la transversalidad, y la introducción, paulatina, del aprendizaje a la carta y los grados abiertos (JIMÉNEZ CARDONA, 26 de octubre de 2020, párrs. 6-7). Todo ello requerirá, en paralelo, no solo la consolidación de la "cultura de la calidad" que, como requisito, precisa de estabilidad normativa en el ámbito de la formación universitaria, sino también tener bien claro que, sin perjuicio de las bondades del aprendizaje virtual que se ha demostrado como una buena solución en los momentos presididos por la compleja gestión de la Covid-19 (y el cual, además, también ha llegado para quedarse en un mundo presidido por internet), lo cierto es que una pantalla de ordenador o una videoconferencia nunca debieran sustituir, íntegramente, las virtudes de la formación y aprendizaje presencial (“cara a cara”).

La universidad del siglo XXI debe seguir siendo un "lugar afectivo" y de "sociabilidad" (VALLESPÍN PÉREZ, 28 de mayo de 2020, párrs. 21-24). Las nuevas tecnologías ya son, como también lo serán en el futuro, una herramienta muy útil a nivel formativo. Sin embargo, no debieran convertirse en un pilar educativo, sino más bien en su imprescindible complemento. De ahí, precisamente, que parezca oportuno combinar las metodologías docentes más clásicas, como la propia clase magistral, todavía de gran utilidad en orden a la transmisión de conocimientos; con aquellas otras de naturaleza 
activa (vgr. aprendizaje basado en problemas, aprendizaje servicio, aula inversa, gamificación y ludificación) $)^{122}$.

La innovación y la calidad más que objetivos, son genuinos requisitos de una docencia de calidad. En este contexto, la universidad debe afrontar, sin más dilaciones, la formación transversal del alumnado sobre la base de los ODS. Las y los estudiantes universitarios, más allá de lo que constituye su formación particular en un determinado ámbito de conocimiento, deben también formarse en relación a su concienciación cívica, medioambiental, ética, humanística y científica. La ignorancia del alumnado universitario acerca de las variadas implicaciones del desarrollo sostenible no es, en consecuencia, una opción, sino más bien un problema que debe solucionarse con urgencia y en función de un claro compromiso intergeneracional.

De otra parte, una de las principales misiones de la universidad debe ser la de generar conocimiento mediante la investigación. Destinar recursos para la investigación es apostar por el futuro de una sociedad. Por tanto, el fomento de una investigación de calidad, tanto básica como aplicada, y en absoluto ajena a la internacionalización, así como tampoco a las líneas de interés social del tercer sector, la protección de los más desfavorecidos, la igualdad, la sostenibilidad y la cooperación; exige desarrollar una política científica dirigida no solo a consolidar y mejorar la máxima competitividad de los grupos de investigación universitarios, sino también a facilitar la promoción del papel de las universidades en el modelo de investigación pública del cual debe dotarse cualquier Estado avanzado.

Todo ello sin olvidar, además, que la propia responsabilidad social que corresponde a la universidad hace que ésta deba procurar, en todo caso, que la investigación acabe por transformarse, en positivo, en bienestar social, económico y cultural. Las universidades tienen un gran potencial para convertirse en centros relevantes en materia de transferencia e innovación, pero tienen que acabar de creérselo. Es hora de apostar, desde la universidad, por un modelo de dinamización de la transferencia e innovación. Un modelo

\footnotetext{
${ }^{122}$ En esta línea de pensamiento, referida a la docencia y el aprendizaje en el ámbito de las ciencias jurídicas, véase: JIMÉNEZ CARDONA, N. (2019). Reflexiones acerca de una experiencia de "gamificación" en Derecho Mercantil dentro del grado de ADE de la Universitat de Barcelona. Revista Educación y Derecho - Education and Law Review, (21), 1-13.
} 
de cuádruple hélice en que se hagan patentes las conexiones recíprocas entre universidad, gobierno, tejido productivo y sociedad. Para ello es necesario conseguir que funcione, de una vez por todas, la relación y colaboración constructiva entre universidad y empresa.

Llegados a este punto, es obligado señalar que los países con mayor equilibrio social son aquellos que disponen de una industria fuerte y competitiva. Apostar, sin perjuicio del papel fundamental que corresponden a las ciencias de la salud y experimentales, por el fomento de la innovación de naturaleza social, es imprescindible. La puesta en marcha de un adecuado plan estratégico de difusión, comunicación y cultura científica (VALLESPÍN PÉREZ, 3 de noviembre de 2020, párrs. 8-10) debe facilitar, de una parte, la apropiación "social” de la ciencia, la tecnología, la cultura emprendedora y la innovación; y, de otra, hacer posible la consolidación del nuevo contrato social al que antes nos hemos referido.

\section{Política medioambiental y universidad}

La universidad, al hilo de los ODS, como no puede ser de otra forma, debe poner en marcha una política activa medioambiental fundada en el desarrollo sostenible, preocupada por el cambio climático y concienciada respecto a la necesidad de incorporar a sus valores la llamada "marca verde", esto es, un claro e inequívoco compromiso para con la ciudadanía y el planeta (FERRER CARBONELL, LAZO MACHO y PIERRA CONDE, 2004, 88-89).

Esta política medioambiental en el ámbito universitario requiere asumir, conscientemente, su propia sostenibilidad. Esta asunción implica, concretamente, el cumplimiento de once grandes compromisos: 1) diseñar un proceso de integración de los ODS que permita identificar cuáles son los temas ya tratados a nivel universitario, identificar prioridades, oportunidades y lagunas, e integrar e implementar diferentes acciones estratégicas de protección del medio ambiente (plan de desarrollo sostenible); 2) optimizar los recursos mediante la búsqueda de la eficiencia y el ahorro en todos los procesos y niveles de actuación, con el consiguiente beneficio económico; 3 ) asumir el compromiso ético y de solidaridad para con la sociedad y, en general, para con la humanidad en su conjunto; 4) respetar los elementos y valores medioambientales, en especial los biológicos, tanto dentro como fuera de la universidad, así como reducir los 
residuos en general; 5) impregnar la formación del alumnado, a todos los niveles académicos, así como la práctica diaria de los cometidos del PDI y del PAS, de una clara concienciación acerca del cumplimiento de los valores propios de la sostenibilidad; 6) fomentar la incorporación de las diferentes perspectivas de la sostenibilidad en las actividades de investigación, transferencia e innovación universitaria; 7) impulsar la transferencia de conocimiento como medio para alcanzar la mejora social; 8) estabilizar y profesionalizar la estructura administrativa de soporte a la sostenibilidad; 9) impulsar las políticas emprendedoras del alumnado con pleno respeto a los ODS; 10) implicar la institución universitaria en la proyección y debates internacionales acerca de la protección del medio ambiente y el cambio climático; y 11) poner en marcha medidas de movilidad sostenible en los propios campus universitarios (por ejemplo, mediante la mejora de los precios del transporte público para los miembros de la comunidad universitaria; o el fomento de la utilización de la bicicleta o de patinetes eléctricos).

Dichos compromisos exigen, transversalmente, que las universidades centren su actuación en los ODS respecto a cuatro grandes pilares: el económico (medidas de ahorro energético, producción de energía mediante la instalación de placas solares, reducción del calentamiento y conductividad térmica de las cubiertas, refuerzo de los aislamientos térmicos, medidas de ahorro de agua); el social (asunción del valor sostenibilidad como propio, elegir las empresas suministradoras en función del seguimiento de parámetros de producción ecológica y de condiciones laborales justas y de proximidad, creación de "comandos verdes" entre el alumnado dirigidos a dinamizar en dicho colectivo hábitos sostenibles, o la adopción de medidas puntuales que permitan la mejora en el ranking GreenMetric); el ambiental en sentido estricto (arborizar y enjardinar los espacios comunes al aire libre, introducir y sustituir algunas especies vegetales de las áreas enjardinadas por otras más adaptadas al clima local, así como eliminar aquellas otras con alto valor alérgico, recoger y gestionar la biomasa producida en las instalaciones universitarias, mantener un calendario correcto de podas, o reforzar el reciclaje); y el educativo (fomentar los contenidos ambientales y de sostenibilidad en los planes de estudio, impulsar contenidos transversales sobre medio ambiente, ecología, cambio climático y sostenibilidad en diferentes asignaturas, crear un laboratorio para impulsar proyectos relacionados con los ODS, y colaborar en campañas educativas para con la ciudadanía y su sensibilización en relación al cambio climático). 
Hecho esto, las universidades estarán en condiciones de incorporar a sus valores corporativos la "marca verde" de su compromiso medioambiental, lo cual, por extensión, ha de facilitar, en paralelo, su acreditación o certificación ambiental de calidad. Ello es así, porque en un contexto de "economía ecológica" en el que se combinen, de una parte, la economía circular, inspirada en la naturaleza y que busca transformar los residuos en nuevos recursos; y, de otra, la economía del bien común, en beneficio no solo de la sociedad actual, sino también de las próximas generaciones; las universidades deben situar en el centro de su actividad las políticas de desarrollo sostenible y medioambiental, no tanto por la modernidad, moda o pedigrí que ello pueda suponer, sino más bien por exigencias que derivan de la necesidad económica y obligación ética para con los congéneres y el planeta (MARTÍN VIDE, 2020, 21-22).

\section{La igualdad efectiva entre mujeres y hombres en el ámbito universitario}

Alcanzar la igualdad en la universidad es un objetivo obligado que exige "mover sus fundamentos". No se trata de un simple retoque estético, sino de un auténtico cambio de paradigma. Es evidente que en el momento presente no partimos de cero, pero todavía queda mucho camino por recorrer para alcanzar una igualdad, realmente efectiva, entre mujeres y hombres en el sistema universitario (ESPEJO MEGÍAS, 2019, 9-11).

En coherencia con lo que constituye hoy el ODS núm. 5 de la Agenda 2030, toda institución universitaria debe asumir un firme compromiso con la igualdad de oportunidades y de trato, así como con la no discriminación. El derecho a la igualdad de oportunidades entre hombres y mujeres es un principio jurídico universal y está plenamente reconocido en diferentes textos internacionales sobre derechos humanos. Es innegable que diferentes normas legales, nacionales, autonómicas e internacionales, contemplan, hoy, el derecho a la no discriminación, así como promueven la igualdad de género.

Este cuerpo normativo ha servido para visualizar una realidad: el sesgo de género (MEGÍAS-BAS, 2020, 15-17) y, por extensión, para sensibilizar tanto a la sociedad como a la comunidad universitaria en orden a poner en marcha diferentes políticas de igualdad entre mujeres y hombres. Por desgracia, este conjunto normativo, todavía a día de hoy, 
no ha conseguido acabar con las desigualdades en el reclutamiento, promoción y retención del personal en nuestras universidades (EGEA RECHE, 2006, 200-201).

Algunos datos concretos reflejados en el Informe de la Xarxa Vives d'Universitats de $2019^{123}$ así lo demuestran: a) las mujeres representan el $42 \%$ del personal docente e investigador de las universidades (PDI); b) a media que aumenta el rango académico del PDI, disminuye el porcentaje de mujeres; c) en idéntica situación profesional y de número de hijos, los hombres publican 1,5 artículos más de media que las mujeres; d) casi el 70\% de los investigadores principales de los diferentes proyectos son hombres; e) solo el $36 \%$ de las mujeres tienen uno o más tramos de investigación; f) las mujeres tienen menos acceso a los puestos de decisión, pero asumen, por regla general, más cargas de gestión; g) siguen siendo las mujeres las que en un $75 \%$ siguen solicitando los permisos para cuidar de los hijos o de las personas mayores dependientes; h) en el colectivo del personal de administración y servicios (PAS) los puestos funcionariales de mayor jerarquía siguen siendo ocupados por hombres; e i) si bien es cierto que ya contamos, dentro del estudiantado universitario, con una mayoría de mujeres (el 54\%), no lo es menos que su número se reduce, proporcionalmente, en función de las diferentes etapas de su carrera académica.

En consecuencia, la universidad no es ajena a la "segregación vertical" que obedece al llamado "techo de cristal" - barreras invisibles que dificultan el acceso de la mujer a los puestos directivos -, así como tampoco a manifestaciones del "suelo enganchoso" constatación de fuerzas que retienen a las mujeres, por regla general, en trabajos de menor relevancia, prestigio o salario (VALLESPÍN PÉREZ, 18 de febrero de 2020, párrs. 4-5).

Siendo esto así, la batalla por la igualdad persiste. Una batalla que debe ser compartida por hombres y mujeres y en la que la universidad, como motor de los cambios sociales y culturales, está llamada a jugar un rol fundamental si, de verdad, queremos construir una sociedad más justa e igualitaria. El hecho de que las instituciones de educación superior hayan dado, en los últimos años, pasos decididos en orden a asegurar la igualdad "formal"

\footnotetext{
${ }^{123}$ Xarxa Vives d'Universitats. (2019). El biaix de gènere en el reclutament, la promoció i la retenció del personal a les universitats. Recuperado de: https://www.vives.org/book/el-biaix-de-genere-en-elreclutament-la-promocio-i-la-retencio-del-personal-a-les-universitats-informe-2019/.
} 
entre mujeres y hombres; no garantiza, per se, que podamos hablar de una igualdad de género realmente efectiva ${ }^{124}$.

Es por ello que las políticas de igualdad deben situarse, por convicción, en el propio corazón de las instituciones universitarias. Se trata de políticas absolutamente transversales y que atraviesan toda la universidad, desde el nivel de la toma de decisiones hasta la economía, la docencia, la investigación, la transferencia de conocimiento e innovación, y las relaciones entre la política y la sociedad civil. En los últimos años son muchas las universidades que en temas de igualdad se mueven dentro de lo "políticamente correcto", lo cual ha derivado en la elaboración de diferentes planes y estructuras de igualdad. Sin embargo, esta realidad no siempre implica la constatación de un auténtico compromiso institucional para con la igualdad de género.

Para conseguir la igualdad efectiva de mujeres y hombres es obligado asumir, con criterios racionales, un enfoque activo de dicha igualdad (gender mainstreaming). Una visión que deberá afectar a los actuales protocolos de acoso sexual, el cambio de nombre de las personas transexuales o transgénero, el asesoramiento en la docencia, la investigación y la transferencia desde la perspectiva de género, la organización de actividades de sensibilización, y la introducción de los temas de género en el ámbito de la formación del profesorado novel. Ello obliga a las universidades a trabajar en una triple dirección: a) promover la presencia equilibrada de mujeres y hombres en los equipos de investigación; b) conseguir la igualdad de género en los diferentes niveles de toma de decisiones; y c) integrar el análisis de la dimensión de sexo/género en el contenido de la investigación específica, con inclusión de la evaluación de los resultados obtenidos.

La política de igualdad de las universidades debiera centrar su foco de atención alrededor de cinco grandes ámbitos concurrentes: gobernanza en igualdad, igualdad en la investigación, docencia en igualdad e igualdad en la docencia, tolerancia cero con la violencia de género y el acoso sexual, y consolidación de una academia compatible con la vida personal y familiar.

\footnotetext{
${ }^{124}$ Sobre este particular, con una visión comparada, véase, por todos: FRUTOS BALIBREA, L. (2019). Desigualdades en educación superior: una mirada desde Europa. Revista Educación y Derecho - Education and Law Review, (19), 1-7.
} 
La gobernanza en igualdad requiere un análisis previo acerca de la cultura institucional, la sensibilización en atención a las desigualdades ya constatables, la promoción de la carrera académica y profesional de las mujeres, la reducción de la segregación vertical y horizontal, y el progreso en cuanto a alcanzar la equidad en los órganos de representación y gobierno. Esta gobernanza requiere contar con planes de igualdad que no lo sean solo institucionales, sino que también incorporen una auténtica regulación de la igualdad en el trabajo y en la vida académica; desglosar los datos por género en docencia, investigación, transferencia y gestión; asegurar la igualdad salarial; respetar el lenguaje inclusivo; diseñar estrategias de promoción académica en el ámbito de la legislación vigente; potenciar la equidad en las comisiones, órganos de gobierno y de representación; y potenciar las acciones y relaciones internacionales en orden a la igualdad.

Por lo que hace referencia a la igualdad en la docencia y la docencia en igualdad, es obligado tener bien presente la necesidad de enfocar la práctica docente, la innovación docente y la investigación en docencia, con una perspectiva de género, así como tomar conciencia acerca de la repercusión desigual de ciertas prácticas docentes en función del género. Serán necesarios cursos de formación en estrategias docentes activas y en igualdad, así como guías para una docencia con perspectiva de género y la potenciación de TFG y TFM sobre perspectiva de género, ya sean específicos de la disciplina o transversales.

La visión de la igualdad en orden a la investigación exige tener una perspectiva histórica que permita visualizar el papel de la mujer en el ámbito de la ciencia, pero también tomar conciencia acerca de la necesidad de desarrollar e incentivar la investigación con perspectiva de género, así como reflexionar sobre el modelo de excelencia en la investigación y la promoción del talento femenino. En esta línea de actuación tienen pleno sentido, entre otras acciones: asesorar acerca de los requisitos vinculados con la perspectiva de género en los proyectos de investigación; sensibilizar sobre los sesgos de género constatables a nivel investigador; promover la equidad en cuanto al liderazgo de grupos, equipos o institutos de investigación; implementar acciones positivas para intensificar la investigación tras la baja maternal; y computar, como tarea del PDI, la participación en comisiones y tribunales, ya que en aras a la deseable paridad no son pocos los casos en los que la mujeres acaban siendo sobrecargadas, sin compensación alguna, en cuanto a su presencia activa en dichas comisiones y tribunales. 
De igual modo, como no puede ser de otra forma, las universidades deberán aplicar una tolerancia cero respecto a la violencia por motivos de género. Para ello es necesario que implementen políticas de prevención, revisen la operatividad de los actuales protocolos sobre acoso, establezcan procedimientos sancionadores diseñados con todas las garantías, y pongan en marcha oficinas de información, prevención y acompañamiento (“puntos lilas").

Respecto a la construcción de una academia compatible con la vida personal y familiar, las universidades tendrán que generar las condiciones óptimas que garanticen el bienestar de las personas, así como las oportunidades que permitan conciliar, de verdad, las exigencias laborales con la vida familiar. Las universidades, en esta línea, tienen el deber inexcusable de asumir su corresponsabilidad mediante la negociación acerca de la racionalización del calendario de las convocatorias competitivas; la creación de guarderías y centros de atención a personas dependientes; y la consolidación de un servicio de atención psicológica gratuita para la comunidad universitaria.

La actuación que se acaba de exponer en torno a los cinco ámbitos referidos para con la igualdad efectiva entre mujeres y hombres requerirá, en paralelo, que las instituciones universitarias activen un conjunto de buenas prácticas en orden a favorecer y conseguir dicha igualdad: a) elaboración y actualización de los planes de igualdad; b) diagnóstico de las discriminaciones o desigualdades entre mujeres y hombres; c) análisis de una docencia presidida por la perspectiva de género, así como de la brecha salarial y de los riesgos de violencia de género; y d) diseño de políticas de investigación y transferencia, así como de políticas presupuestarias, claramente fundadas en la perspectiva de género.

\section{Universidad integradora, saludable e implicada en la gestión de la diversidad y la cohesión social}

La universidad sostenible debe ser también una universidad integradora, inclusiva, saludable e implicada en la gestión de la diversidad y la cohesión social. Integradora e inclusiva, porque debe representar la voluntad de la institución para promover espacios de integración de las personas con discapacidad mediante la concienciación y el pleno 
respeto de la accesibilidad universal y la autonomía personal ${ }^{125}$. Una universidad, en fin, que potencie las actividades de atención social; promueva planes específicos de atención personalizada a la comunidad universitaria con discapacidad; ponga en marcha un amplio abanico de medidas vinculadas con aquellas personas con diversidad funcional mediante la promoción de aquellas condiciones que hagan posible que todas ellas puedan desarrollarse a nivel intelectual, profesional y humano, sin ningún tipo de discriminación; y preste especial atención a las personas mayores (vgr. Universidad de la Experiencia).

En esta línea de actuación, la universidad debe involucrarse, por convicción, en los programas de "Unidiversidad". De ese modo, debe dar soporte activo, por ejemplo, a aquellas iniciativas de inserción laboral para jóvenes con discapacidad intelectual, a aquellos programas de convivencia intergeneracional dirigidos no solo a mejorar la calidad de vida de las personas mayores, sino también la de las y los jóvenes estudiantes universitarios (intercambio solidario y no lucrativo basado en la convivencia entre dos generaciones y que persigue, de una parte, que las personas mayores que viven solas puedan mantenerse en su vivienda habitual, evitando así situaciones de deterioro personal y de aislamiento social; y, de otra, la mejora de las condiciones de vida de los estudiantes universitarios que deben hacer frente a importantes desembolsos económicos, no accesibles para todas las familias, en orden a gozar de una alternativa de alojamiento en las grandes ciudades); y a la promoción, dentro de las estrategias más amplias de salud sexual y reproductiva, de la formación en sexualidad y sexología clínica en diferentes grados, así como de la mejora de la atención de la población universitaria en general.

Universidad que también, de conformidad con los ODS, debe ser saludable. Más allá de las acciones relacionadas con la prevención y gestión de la Covid-19, así como con la necesidad de asegurar la seguridad de los edificios, laboratorios y demás instalaciones universitarias; resulta obligado, en pleno siglo XXI, que la universidad también asuma un importante rol en cuanto a la concienciación acerca de la necesidad de mejorar nuestros hábitos de vida (CERRILLO BORJA, MARTÍNEZ RIERA y LÓPEZ GÓMEZ, 2019, 11). Entre otras acciones, la universidad debe implicarse con la potenciación de la

${ }^{125}$ Con carácter monográfico y en relación a lo que supone una universidad inclusiva, véase, por todos: GARCÍA-CANO TORRICO, M., BUENESTADO FERNÁNDEZ, M., GUTIÉRREZ ARENAS, P., LÓPEZ GONZÁLEZ, M. y NARANJO DE ARCOS, A. (2017). Apuntes para la inclusión en la comunidad universitaria. ¿Qué es una Universidad inclusiva? Córdoba: Colección Diversidad - Universidad de Córdoba, 14-24. 
actividad física y el deporte, el fomento de una alimentación saludable, sana y equilibrada (prevención de enfermedades y mejora de la salud), y la mejora de la gestión propia de los riesgos laborales.

Finalmente, una universidad sostenible también debe estar plenamente implicada con la gestión de la diversidad y la cohesión social ${ }^{126}$. Una universidad que sea capaz de fomentar el diálogo entre culturas, la calidad de vida y el bienestar social, así como de luchar contra cualquier tipo de discriminación étnica o social, situar el papel de la mujer en el centro del cambio social (perspectiva de género), diseñar un pacto que facilite la convivencia y garantice el futuro de las próximas generaciones, y actuar como auténtico motor de la gestión del talento y la cultura emprendedora a nivel social.

\section{Conclusiones}

Primera.- La universidad del siglo XXI debe asumir un claro compromiso con los Objetivos de Desarrollo Sostenible de la Agenda 2030. Algunos de dichos objetivos, como el núm. 4, referido a la Educación de Calidad, tiene una implicación directa con las misiones universitarias: docencia, investigación y transferencia e innovación; pero lo cierto es que la universidad, mediante sus políticas, también contribuye, indirectamente, en orden a garantizar el respeto del resto de ODS.

Segunda.- El libre acceso a la universidad pública, entendido éste no como un privilegio, sino como un derecho, tiene que ver con el papel que corresponde a las instituciones de educación superior como garantes del "ascenso social”. Un libre acceso que, en el momento presente, también debe contemplar las medidas oportunas de corrección en orden a la denominada "brecha digital".

Tercera.- La universidad debe adaptarse, como siempre ha hecho a lo largo de la historia, a las nuevas exigencias sociales. De ahí, precisamente, que la universidad, en el ámbito propio de la construcción de la sociedad y economía del conocimiento, deba jugar un

\footnotetext{
${ }^{126}$ Sobre la gestión de la diversidad y la cohesión social y sus implicaciones educativas (también a nivel universitario), véase, por todos: GONZÁLEZ GERALDO, J. L. (2015). Educación, desarrollo y cohesión social. Castilla-La Mancha: Colección Estudios 150, Ediciones de la Universidad de la UCLM.
} 
papel protagonista en orden al diseño e implementación de un nuevo contrato social sustentado, en buena medida, en los ODS de la Agenda 2030.

Cuarta.- Las instituciones de educación superior, al amparo del debido respeto al ODS 4, deberán apostar, decididamente, por la "cultura de la calidad" en el cumplimiento de sus misiones. La innovación y la calidad son requisitos de una docencia de calidad. La universidad debe seguir generando conocimiento mediante la investigación, así como transformar sus resultados, mediante la transferencia e innovación, en bienestar social, económico y cultural.

Quinta.- La universidad debe poner en marcha una política medioambiental fundada en el desarrollo sostenible y la atención al cambio climático, así como concienciada, fruto de su compromiso intergeneracional, para con el bienestar de la ciudadanía y la protección del planeta ("marca verde"). Para ello, desde la perspectiva de los ODS, centrará su acción concurrente en cuatro grandes pilares: el económico, el social, el medioambiental, y el educativo-cultural.

Sexta.- En coherencia con lo que constituye el ODS 05, toda institución universitaria debe asumir un firme compromiso, por convicción, con la igualdad de oportunidades y de trato, así como con la no discriminación. La universidad debe prestar atención a la puesta en marcha de todas aquellas medidas que permitan conseguir una igualdad efectiva entre mujeres y hombres. Para conseguirlo, es oportuno que las instituciones de educación superior centren su atención en diferentes ámbitos concurrentes: el gobierno en igualdad, la docencia en igualdad y la igualdad en la docencia; la perspectiva de género en el ámbito de la investigación y la transferencia e innovación, la tolerancia cero con el acoso sexual y la violencia de género, y el diseño de una academia compatible con la vida personal y familiar.

Séptima.- Finalmente, para poder hablar de una universidad sostenible es obligado que ésta sea también, a la luz de la Agenda 2030, una universidad integradora e inclusiva, saludable, y claramente implicada con la eficaz gestión de la diversidad y la cohesión social. 


\section{Bibliografía}

ANDRÉS AUCEJO, E. (2012). La formación inicial de Jueces y Magistrados: nuevas metodologías de aprendizaje durante el período de formación en la Escuela Judicial. Revista Educación y Derecho - Education and Law Review, (6), 1-7.

CERRILlO BORJA, M., MARTÍNEZ RIERA, J.R. y LÓPEZ GOMEZ, J. (2019). El movimiento de Universidades Saludables en España. Revista Iberoamericana de Enfermería Comunitaria, 12 (1), 9-33.

COSTAS, A. (2017). El final del desconcierto. Un nuevo contrato social para que España funcione. Madrid: Atalaya.

EGEA RECHE, M. (2006). La igualdad en la educación superior: ¿mito o realidad?. Cuestiones de Género, (1), 195-203.

ESPEJO MEGÍAS, P. (2019). Mujeres y Universidad: situación actual y algunas propuestas para el cambio. Revista Educación y Derecho - Education and Law Review, (20), 1-24.

FACHELLI, S. (2019). El rol de la educación superior en la movilidad ocupacional intergeneracional: análisis comparado entre Argentina y España. Revista Educación y Derecho-Educations and Law Review, (19), 1-27.

FERRER CARBONELL, E., LAZO MACHADO, J. y PIERRA CONDE, A. (2004). Universidad y Desarrollo Sostenible. Revista de Pedagogía Universitaria, 9 (3), 86-95.

FRUTOS BALIBREA, L. (2019). Desigualdades en educación superior: una mirada desde Europa. Revista Educación y Derecho - Education and Law Review, (19), 1-7.

GARCÍA, I. y ASTIGARRAGA, E. (1 de mayo de 2020). Los objetivos de desarrollo sostenible en la formación universitaria. Blog de Studia XXI - Universídad. Recuperado de: https://www.universidadsi.es/objetivos-de-desarrollo-sostenible-universidad/ 
GARCÍA BENAU, Mª A. (14 de enero de 2019). Objetivos de desarrollo sostenible: nuevos retos y oportunidades para las universidades. Blog de Studia XXI - Universídad. Recuperado de: https://www.universidadsi.es/objetivos-de-desarrollo-sostenible-nuevosretos-y-oportunidades-para-las-universidades/

GARCÍA-CANO TORRICO, M., BUENESTADO FERNÁNDEZ, M., GUTIÉRREZ ARENAS, P., LÓPEZ GONZÁLEZ, M. y NARANJO DE ARCOS, A. (2017). Apuntes para la inclusión en la comunidad universitaria. ¿Qué es una Universidad inclusiva? Córdoba: Colección Diversidad - Universidad de Córdoba.

GONZÁLEZ GERALDO, J. L. (2015). Educación, desarrollo y cohesión social. CastillaLa Mancha: Colección Estudios 150, Ediciones de la Universidad de la UCLM.

Informe de Xarxa Vives d'Universitats. (2019). El biaix de gènere en el reclutament, la promoció i la retenció del personal a les universitats. Recuperado de: https://www.vives.org/book/el-biaix-de-genere-en-el-reclutament-la-promocio-i-laretencio-del-personal-a-les-universitats-informe-2019/

JIMÉNEZ CARDONA, N. (2019). Reflexiones acerca de una experiencia de gamificación en Derecho Mercantil dentro del grado de ADE de la Universitat de Barcelona. Revista Educación y Derecho - Education and Law Review, (21), 1-13.

JIMÉNEZ CARDONA, N. (26 de octubre de 2020). ¿Vale la pena estudiar un doble grado o un grado abierto?. Blog de Studia XXI- Universídad. Recuperado de: https://www.universidadsi.es/vale-la-pena-estudiar-dobles-grados-o-un-grado-abierto/

MARTÍN VIDE, J. (2020). Risc global, solución urgent. Serra d'Or, (724), 18-23.

MEGÍAS-BAS, A. (2019). Sesgos de género en la Educación Superior en España: propuestas de actuación. Revista Educación y Derecho - Education and Law Review, (20), 1-24.

MONEVA ABADÍA, J.M. y MARTÍN VALLESPÍN, E. (2012). Universidad y Desarrollo Sostenible: análisis de la rendición de cuentas de las universidades públicas 
desde un enfoque de responsabilidad social. Revista iberoamericana de contabilidad de gestión, 10 (19), 1-19.

OWENS, J., LENNARD, M. y ANDRÉS AUCEJO, E. (2020). La financiación del Desarrollo Sostenible: Tributación y Objetivos de Desarrollo Sostenible. Policy-making en Tributación, Cooperación tributaria internacional y Gobernanza Fiscal Mundial como principal fuente de financiación de la Agenda 2030 de las Naciones Unidads (I Parte). Revista Educación y Derecho - Education and Law Review, (21), 1-16.

RODRÍGUEZ LOZANO, P. (23 de junio de 2020). Las universidades como líderes en la implementación de los ODS. Blog de Studia XXI - Universídad. Recuperado de: https://www.universidadsi.es/las-universidades-como-lideres-en-la-implementacionde-los-ods/

SUREDA, J., SÁNCHEZ, F. y BENAYAS, J. (9 de junio de 2017). Sostenibilidad de las universidades y objetivos de desarrollo sostenible de Naciones Unidas. Blog de Studia Siglo XXI - Universídad. Recuperado de: https://www.universidadsi.es/sostenibilidad-las-universidades-objetivos-desarrollosostenible-naciones-unidas/

VALLESPÍN PÉREZ, D. (18 de octubre de 2016). L'educació superior, un dret i no un privilegi. Nació Digital. Recuperado de: https://www.naciodigital.cat/opinio/13955/educacio-superior-dret-no-privilegi

VALLESPÍN PÉREZ, D. (29 de mayo de 2018). La transformación de la universidad pública. $\quad$ El País. Recuperado de: https://elpais.com/elpais/2018/05/18/opinion/1526655439_062635.html

VALLESPÍN PÉREZ, D. (3 de julio de 2018). Finançament universitari. El Punt Avui. Recuperado de: https://www.elpuntavui.cat/opinio/article/8-articles/1424394financament-universitari.html 
VALLESPÍN PÉREZ, D. (17 de junio de 2019). Desarrollo sostenible, biogeología y universidad. Blog de Studia XXI - Universídad. Recuperado de: https://www.universidadsi.es/desarrollo-sostenible-biogeologia-y-universidad/

VALLESPÍN PÉREZ, D. (24 de octubre de 2019). Fundraising y alumni: dos elementos a tener en cuenta en la financiación de las universidades públicas. Blog de Studia XXI - Universídad. Recuperado de: https://www.universidadsi.es/fundraisingy-alumni-dos-elementos-a-tener-en-cuenta-en-la-financiacion-de-la-universidadespublicas/

VALLESPÍN PÉREZ, D. (18 de febrero de 2020). El sesgo de género en la universidad. Blog de Studia XXI - Universídad. Recuperado de: https://www.universidadsi.es/el-sesgo-de-genero-en-la-universidad/

VALLESPÍN PÉREZ, D. (15 de marzo de 2020). La revolució digital. El Punt Avui. Recuperado de: https://www.elpuntavui.cat/opinio/article/1757436-la-revoluciodigital.html

VALLESPÍN PÉREZ, D. (21 de mayo de 2020). La Universidad ante el nuevo contrato social. elDiario.es. Recuperado de: https://www.eldiario.es/opinion/tribunaabierta/universidad-nuevo-contrato-social_129_5962112.html

VALLESPÍN PÉREZ, D. (28 de mayo de 2020). La nueva normalidad docente en la universidad. Blog de Studia XXI - Universídad. Recuperado de: https://www.universidadsi.es/la-nueva-normalidad-docente-en-la-universidad/

VALLESPÍN PÉREZ, D. (5 de octubre de 2020). La UB del segle XXI. El Periódico de Catalunya. Recuperado de: https://www.elperiodico.cat/ca/opinio/20201005/article-david-vallespin-universitatbarcelona-segle-xxi-8142398

VALLESPÍN PÉREZ, D. (3 de noviembre de 2020). La divulgación científica como estrategia universitaria. Blog de Studia XXI- Universídad. Recuperado de: https://www.universidadsi.es/divulgacion-cientifica-estrategia-universitaria/ 